\title{
Éléments de réussite économique des élevages ovins extensifs du Montmorillonnais
}

\author{
Marc Benoit ${ }^{a *}$, Gabriel Laignela ${ }^{\mathfrak{a}}$, Gilbert Liénard ${ }^{\mathrm{a}}$, Benoît Dedieu ${ }^{\mathrm{b}}$, \\ Jean-Marc Chabosseau ${ }^{\mathrm{c}}$
}
'Laboratoire d'économie de l'élevage, Inra Theix, 63122 Saint-Genès-Champanelle, France
b SAD et LAHM, Inra Theix, 63122 Saint-Genès-Champanelle, France
' SAPF, Inra, 86600 Lusignan, France

(Reçu le 24 avril 1998 ; accepté le 10 juillet 1998)

\begin{abstract}
Ways to have good economic results in Montmorillonnais's extensive sheep farms. This study demonstrates the special character of extensive farms located in the midst of large meat sheep farms in Montmorillonnais (an unfavorised zone south of Vienne, France). Characteristic production systems are found in this region, from extensive (with a stocking rate of less than $0.85 \mathrm{LU} \cdot \mathrm{ha}^{-1}$ PFS) to intensive (1.40 LU $\cdot \mathrm{ha}^{-1}$ PFS). There are large differences between these systems, even between the various extensive systems among which two sub-groups can be distinguished. The first include extremely autonomous extensive specialists and second are extremely extensive systems with crops. Some intensive farmers make a decent net income as do some grazers and specialised extensive farmers. The three essential factors are the margin per ewe, the work productivity and the infrastructure costs. On extensive farms, the best results are found for moderate numerical productivity (1.15-1.30 lamb per ewe), which make maximal use of grazing and minimal amount of input. The animals spend more time in pasture, reducing the amount of hay needing to be harvested. Some extensive farms obtain good results on small usable agricultural area if they have a high degree of forage autonomy and if their infrastructure costs are limited. $($ Elsevier/Inra
\end{abstract}

\section{sheep / system / extensive / economy}

Résumé - L'étude montre la spécificité d'élevages extensifs au sein d'un groupe de grands élevages ovins viande du Montmorillonnais (zone défavorisée du sud de la Vienne) regroupés en types d'exploitations très caractérisés, des extensifs (avec un chargement inférieur à 0,85 UGB ha $^{-1}$ SFP) à des intensifs $\left(1,40 \mathrm{UGB} \cdot \mathrm{ha}^{-1}\right)$, relativement aux conditions du milieu. Les fonctionnements sont très différents, y compris au sein des extensifs, parmi lesquels on distingue deux sous-groupes, les extensifs spécialisés très autonomes et les très extensifs avec cultures. De bons niveaux de revenu s'observent tant chez certains élevages intensifs que dans les élevages herbagers autonomes ou extensifs spé-

\footnotetext{
* Correspondence and reprints

Tél. : (33) 04736241.34 ; fax : (33) 0473624450 ; e-mail : benoit@clermont.inra.fr
} 
cialisés, en fonction des niveaux des trois facteurs essenticls que sont la marge par brebis, la productivité du travail et les charges de structure. En élevage extensif, les meilleurs résultats reposent sur une productivité numérique modérée ( 115 à $130 \%$ ) avec une utilisation maximale de l'herbe et très peu d'intrants, la pratique du plein air pouvant réduire les stocks fourragers. Certains obtiennent de bons revenus sur des surfaces moyennes, s'ils ont une excellente autonomie fourragère et des charges de structure très limitées. (-) Elsevier/Inra

ovin / allaitant / système / extensif / économie

\section{INTRODUCTION}

Les conditions économiques du développement de l'élevage ovin en France ont profondément changé avec la mise en place du règlement communautaire en 1980 , qui a mis en concurrence les élevages français avec ceux de Grande-Bretagne et d'Irlande. Précédemment « festive et chère », la viande ovine s'est peu à peu banalisée sous l'effet de la baisse des prix qui en a accru la consommation. Une part croissante a été fournie par les importations (de $20 \%$ en 1980 à plus de $50 \%$ à partir des années 1990). Les systèmes d'élevage très intensifs ont été mis en difficulté [1]. De nouvelles conceptions d'un élevage ovin basé sur l'utilisation de plus d'herbe et d'espace ont progressivement émergé - en parallèle à ce qui se réfléchissait pour les bovins et les possibilités d'extensification [4]. Encore fallait-il asseoir les nouvelles perspectives pour l'élevage ovin sur des résultats économiques d'exploitations.

L'étude est réalisée sur la base d'une typologie d'exploitations ovines du Montmorillonnais. Celle-ci illustre la diversité des stratégies mises en place par les éleveurs. Des questions spécifiques concernent les élevages ayant les chargements les plus bas : peuvent-ils atteindre les objectifs de la filières tout en utilisant le maximum de ressources fourragères? Les principaux facteurs du revenu identifiés en élevage ovin allaitant, sur un plan général, - marge brute par brebis, productivité du travail et charges de structure - ont-ils la même importance relative? La marge par brebis peut-elle être maintenue, voire améliorée comme le suggèrent les expérimentations ayant comparé des conduites classiques et des conduites d'extensification avec agrandissement [5, 8]?

\section{BASE DE L'ÉTUDE}

L'étude porte sur les résultats des données 1995 de 38 exploitations. Les surfaces varient de 60 à 280 ha SAU, avec une moyenne de 137 ha. Les chargements fluctuent également fortement, de 0,60 à 1,50 UGB.ha' ${ }^{-1}$ SFP (figure l).

La typologie élaborée est basée sur la prise en compte simultanée des critères de main d'œuvre, de répartition de la surface entre fourrages et cultures, de conduite du troupeau ovin, d'importance des intrants et du degré d'autonomie fourragère, de présence éventuelle de bovins et du niveau des charges de structures. Elle fait apparaître dix groupes (tableau $I$ ) dont les caractéristiques ont été publiées $[2,3,6,7]$ et parmi lesquels on distinguera dans cet exposé :

- des exploitations extensives très autonomes ( $n=3$; chargement moyen de 0,83 ) de 77 à 206 ha, avec un atelier ovin conduit en très forte autonomie fourragère, et un bon niveau de marge par brebis ; les cultures de vente sont absentes ;

- des exploitations très extensives avec cultures $(n=6)$ de très grandes dimensions (224 ha de moyenne) ayant des chargements très faibles $\left(0,72 \mathrm{UGB} \cdot \mathrm{ha}^{-1} \mathrm{SFP}\right)$ dans les- 
Figure 1. Relation entre la surface par travailleur et le chargement selon les types d'exploitations (HA, herbagers autonomes; $I$, intensifs; $E$, extensif).

quelles l'atelier ovin, peu performant, est conduit en parallèle à un secteur de cultures conséquent (64 ha) ; les salariés sont fréquents $(0,50$ en moyenne).

\section{ILLUSTRATION \\ DE DIFFÉRENTES STRATÉGIES}

Plusieurs groupes typologiques révèlent la présence d'exploitations que l'on peut considérer comme performantes au vu de leur revenu par UTH. Ces exploitations se caractérisent par leur cohérence, au plan de leur stratégie globale (combinaison des facteurs essentiels du revenu, marge par brebis, productivité du travail, niveau des charges de structure), et au travers d'une logique de fonctionnement du troupeau ovin tirant au mieux parti du contexte de l'exploitation : disponibilité fourragère (chargement), existence de céréales autoproduites, voire présence d'un troupeau bovin allaitant complémentaire.

Certaines exploitations extensives du réseau mettent en cuuve de nouvelles stra-

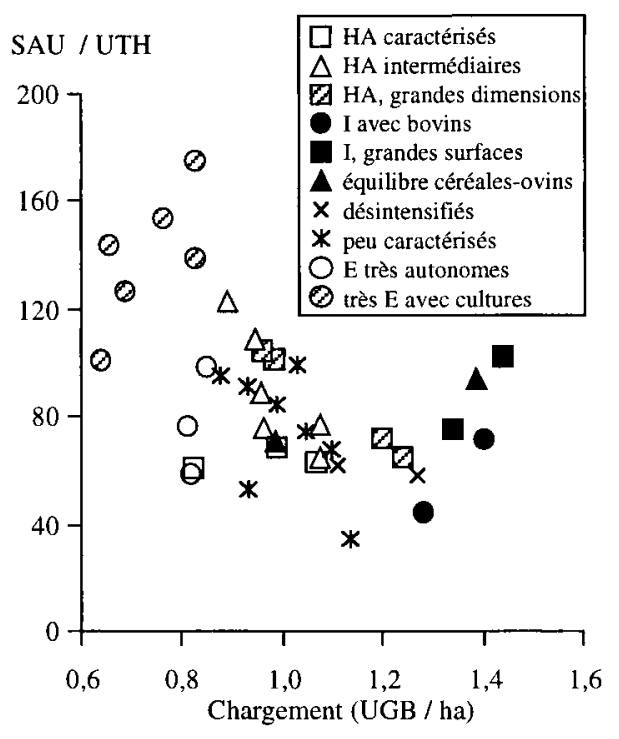

tégies et logiques de fonctionnement de troupeau qui montrent des adaptations originales à des chargements faibles et à ses conséquences.

Un exemple extrême provient d'une exploitation de dimension inférieure à la moyenne (77 ha, 364 brebis et 1 UTH) dont le principe général est l'autonomie maximale pour l'atelier ovin et la limitation des charges de structure qui permettent, malgré un produit par brebis et une productivité du travail inférieurs à la moyenne, d'obtenir un revenu de très bon niveau $(170000 \mathrm{~F} /$ UTH).

Un autre exemple est issu d'une exploitation de grande dimension ( 175 ha et 847 brebis, 1 UTH, $[3,6,7])$ dont la priorité est donnée au suivi du troupeau (réussite de la reproduction et qualité des agneaux vendus). Le maximum de tâches de mécanisation (nettoyage de parcelles...) est réalisé par service extérieur. L'effet multiplicateur de l'effectif conduit à une marge de l'atelier approchant $560000 \mathrm{~F}$. Le revenu de l'atelier est exceptionnel (près de $300000 \mathrm{~F}$ pour I UTH). 


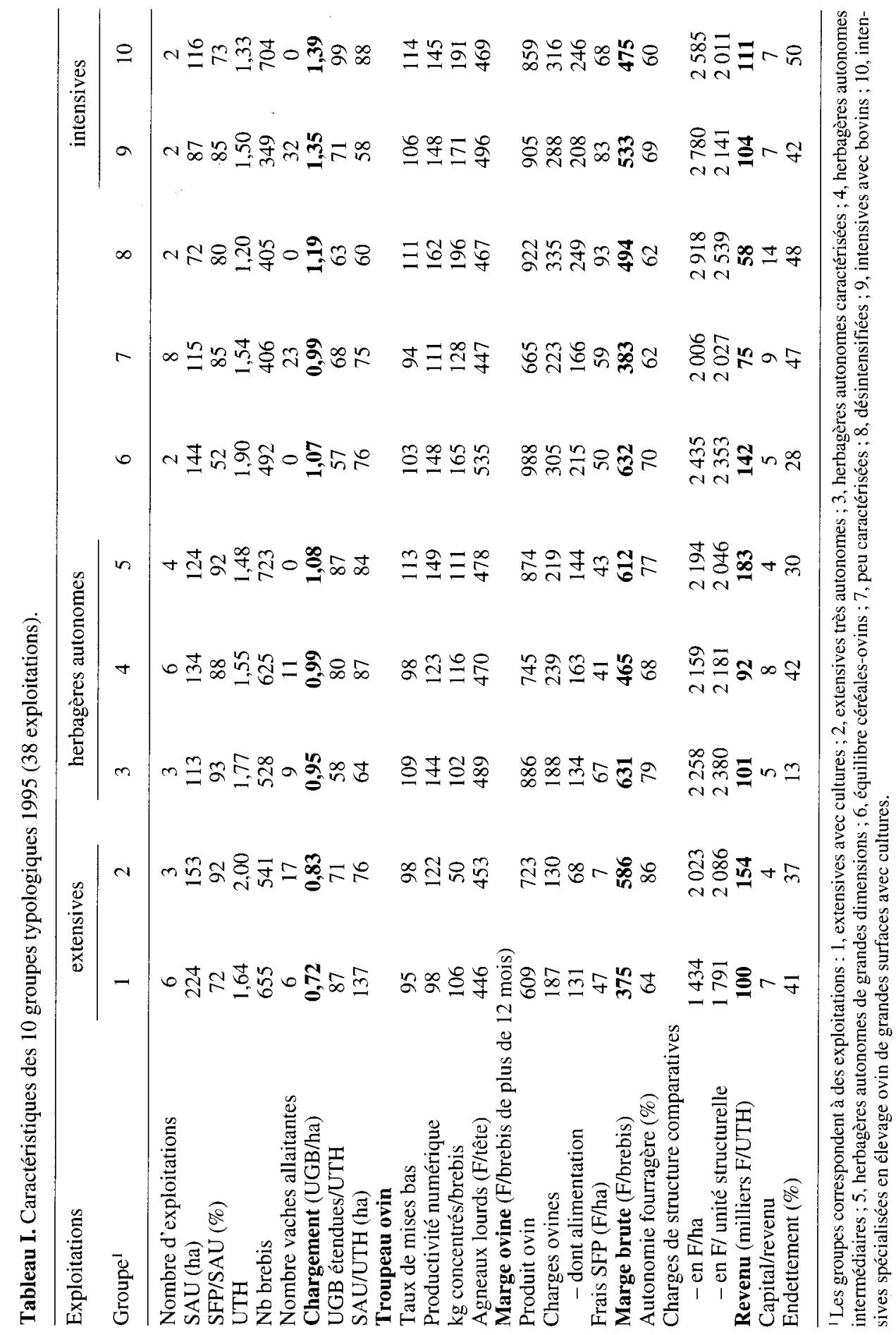




\section{CONDUITES DES ATELIERS OVINS}

Trois paramètres sont dominants dans le résultat économique de l'atelier ovin : la productivité numérique (PN), le coût de l'alimentation directe (consommation de concentrés : $\mathrm{CC}$ ) et, dans une moindre mesure, la valorisation des agneaux. Ils interviennent le plus souvent de façon antagoniste sur la marge brute :

- une PN élevée entraîne des charges d'alimentation plus importantes (accélération des mises bas, agnelages en contre-saison ; prolificité élevée avec complémentation des mères et allaitement artificiel) ;

- une meilleure valorisation des agneaux correspond le plus souvent à les produire en contre-saison, avec des charges d'alimentation plus importantes.

Figure 2. Relation entre marge brute ( $F /$ brebis) et la consommation de concentrés ( $\mathrm{kg} /$ brebis) selon les types d'exploitations (HA, herbagers autonomes ; I, intensifs ; E, extensif).

\subsection{Relation productivité numérique - consommation de concentrés}

La consommation de concentré (agneaux + brebis) rapportée à la brebis varie de 10 à $220 \mathrm{~kg}$. D'excellentes marges par brebis ( 600 à $660 \mathrm{~F} / \mathrm{brebis})$ sont obtenues quel que soit le niveau de concentrés consommés. Pour une même marge de $600 \mathrm{~F}$, la conduite de l'atelier ovin est très variable.

Ainsi, sur le segment [A, B] de la figure 2, le premier éleveur (extensif très autonome, chargement 0,81 ) affiche une marge de $605 \mathrm{~F}$ avec $10 \mathrm{~kg}$ de CC et une PN de $129 \%$. Il a centré toutes les mises bas au printemps. La vente des agneaux, engraissés à l'herbe, s'échelonne de juin à novembre, les derniers étant très mal valorisés car non « finis » et vendus en mauvaise période. L'absence de charges compense cette faible valorisation, l'autonomie fourragère atteint $93 \%$ et

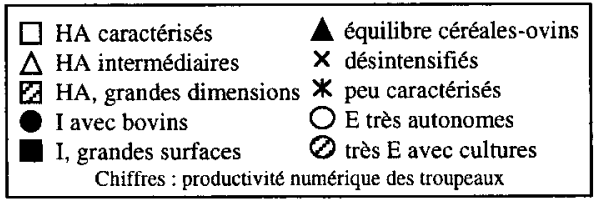

Marge brute ( $F$ / brebis)

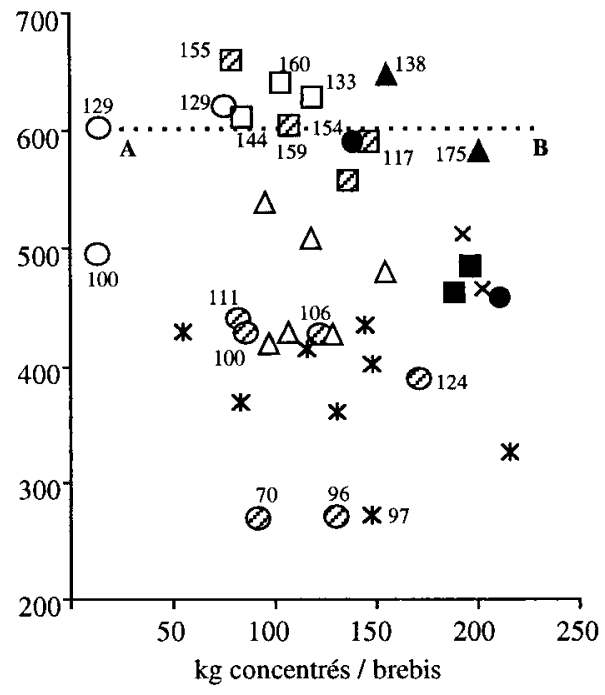


le montant des charges proportionnelles (y compris fourragères) est de $92 \mathrm{~F} / \mathrm{brebis}$.

Le dernier éleveur sur cette ligne de marge à $600 \mathrm{~F} / \mathrm{Br}$ arrive à ce résultat avec une $\mathrm{CC}$ de $200 \mathrm{~kg}$ et une PN de $175 \%$. Céréalier, il a un chargement élevé $\left(1,40 \mathrm{UGB} \cdot \mathrm{ha}^{-1}\right)$, et pratique des agnelages en système accéléré, avec une part importante en contre-saison. La forte consommation de concentrés limite l'autonomie fourragère à $52 \%$.

Les plus faibles marges (trois cas), entre 250 et $300 \mathrm{~F}$ par brebis, résultent d'inadéquation des niveaux de charges et de produit (PN).

\subsection{La notion d'autonomie fourragère}

Parmi les consommations de concentrés les plus faibles, on ne retrouve que des élevages extensifs avec des autonomies fourragères exceptionnelles. Mais une autonomie fourragère élevée n'est pas le fait des seuls extensifs ; elle n'est d'ailleurs pas liée au chargement, même si les deux élevages les plus autonomes ont des chargements de 0,85 UGB $\cdot \mathrm{ha}^{-1}$ SFP. En effet, les élevages herbagers autonomes caractérisés, et grands, avec des conduites fourragères spécifiques performantes (renouvellement des prairies tous les 5 ans, enrubannage éventuel, pression de pâturage forte, assez peu d'azote minéral) obtiennent des autonomies fourragères de 78 et $79 \%$, contre $69 \%$ pour la moyenne de l'échantillon.

À même autonomie fourragère les élevages extensifs ont des produits par brebis plus faibles que les élevages herbagers : la productivité numérique est moindre et le prix des agneaux est souvent inférieur.

\section{REVENUS ET DÉTERMINANTS}

Le revenu du travail et des capitaux propres d'exploitation par travailleur (calculé avec les charges de structures comparatives, foncières et du travail) varie de $27000 \mathrm{~F}$ à 200000 F. Globalement, il apparaît indé- pendant du chargement puisque, entre 0,80 et 1,40 UGB $\cdot \mathrm{ha}^{-1} \mathrm{SFP}$, on retrouve la même dispersion des revenus. En revanche il y a des différences importantes entre les dix types d'exploitations - dont on peut mieux comprendre les raisons après l'analyse des principaux facteurs du revenu.

Pour l'ensemble des 38 exploitations, le premier facteur explicatif de la dispersion des revenus est la marge par brebis $(r=0,48)$, qui dépend très fortement de l'autonomie fourragère $(r=0,72)$ mais aussi de la productivité du troupeau $(r=0,69)$. Ceci explique le fait de retrouver, pour un même revenu, des conduites de troupeaux très différentes.

Le second facteur est, naturellement, la productivité du travail exprimée en nombre d'UGB étendues par travailleur (UGB herbivores $+1 / 2$ surface (ha) des cultures + équivalent hors-sol [2]). Cette productivité varie de 35 à 125 . Mais la relation avec le revenu est nettement plus faible $(r=0,39)$ que celle entre le revenu et la marge. Certes, en dessous de 60 UGB étendues/UTH les revenus sont inférieurs à la moyenne, mais au-dessus, le revenu varie du simple au double pour une même productivité. Les exploitations extensives très autonomes et herbagères autonomes caractérisées obtiennent un excellent revenu avec une productivité du travail moyenne.

Le troisième facteur est constitué des charges de structure comparatives (même statut du foncier et de la main d'œuvre entre exploitations), exprimées par Unité Structurelle (Ustr $=$ UGB + ha de culture + équivalent hors sol). Les valeurs observées varient de 1250 à $2700 \mathrm{~F} /$ Ustr. À partir de $2400 \mathrm{~F} / \mathrm{Ustr}$, elles permettent très difficilement d'obtenir plus de $100000 \mathrm{~F}$ de revenu par UTH. La relation avec le chargement est devenue quasi-inexistante. En revanche les charges foncières comparatives/Ustr sont plutôt supérieures dans les exploitations les moins chargées $(r=-0,37)$ : aux extrêmes, elles varient de $600 \mathrm{~F}$ pour un chargement de 0,70 , à $500 \mathrm{~F}$ pour un 
chargement de 1,30. Ce surcoût de $100 \mathrm{~F} / \mathrm{Ustr}$ doit trouver une compensation - soit sur les autres charges de structures (équipement notamment) - soit par un gain de marge par hectare qui peut provenir d'une marge par UGB meilleure - et/ou de l'obtention de la prime à l'herbe sur les hectares supplémentaires mobilisés, à nombre d'UGB identique.

Les analyses précédentes permettent de comprendre comment se forme le revenu des différents types d'exploitations et les raisons des écarts importants constatés (tableau I). Les meilleurs revenus sont observés dans les systèmes extensifs très autonomes $(154000 \mathrm{~F})$, herbagers autonomes grands (183000 F/UTH), et ovinscéréales (142 000 F/UTH). Les systèmes très extensifs avec cultures, intensifs moyens (ovins-bovins), intensifs grands et herbagers autonomes caractérisés obtiennent des revenus proches de la moyenne (100 à 111000 F/UTH). Les systèmes herbagers moins autonomes, les peu caractérisés et désintensifiés ont les revenus les plus faibles (92000, 75000 et $58000 \mathrm{~F} / \mathrm{UTH})$.

En ce qui concerne les deux groupes extensifs :

- Le revenu est excellent dans les systèmes extensifs très autonomes. Les ateliers ovins, conduits avec des autonomies fourragères comprises entre 84 et $93 \%$, dégagent une marge de $586 \mathrm{~F}$ par brebis, supérieures de $100 \mathrm{~F}$ à la moyenne, alors que le produit par brebis est inférieur de $40 \mathrm{~F}$. Malgré une productivité du travail inférieure à la moyenne (71 UGB étendues/UTH contre 74), ce niveau de marge est déterminant dans la constitution du revenu.

- Les performances de l'atelier ovin des systèmes très extensifs avec cultures sont médiocres, avec une productivité du troupeau de $98 \%$ seulement, associée à une autonomie fourragère de $64 \%$ et à une marge par brebis de $375 \mathrm{~F}$, inférieure de $110 \mathrm{~F}$ à la moyenne. Leur atelier cultures, de 64 ha (dont 22 ha d'oléagineux), n'assure que $35 \%$ de la marge brute globale car la marge à l'hectare est également faible (2 $753 \mathrm{~F} /$ ha ; rendement de 26,5 quintaux par ha de céréales). Deux éléments compensent en partie ces faibles performances : d'une part la productivité du travail est relativement élevée avec 87 UGB étendues/UTH d'autre part les charges de structure sont modérées ( 1791 F/Ustr). Globalement cependant, les faibles performances techniques restent déterminantes puisque le revenu du travail et capitaux par UTH n'atteint que les $2 / 3$ de celui des extensifs très autonomes, avec un niveau par ha de SAU visiblement insuffisant $(730 \mathrm{~F} / \mathrm{ha}$ contre 2010 ).

\section{DISCUSSION}

\subsection{Relations entre le chargement et la conduite du troupeau}

Le suivi de 38 élevages couvrant des situations de chargement de 0,60 à 1,50 UGB $\cdot h^{-1}$ montre que seuls trois élevages ont des autonomies fourragères très fortes (84 à $93 \%$ ). Dans les trois cas, il s'agit d'exploitations extensives dont les chargements sont compris entre 0,80 et $0,85 \mathrm{UGB} / \mathrm{ha}$ SFP.

Dans de telles situations, bien gérées, la disponibilité en surface et ressources fourragères n'est pas limitante et peut assurer l'essentiel de l'alimentation du troupeau et l'engraissement des agneaux à l'herbe. L'adéquation entre les besoins du troupeau et les ressources peut être optimisée, à condition de disposer en permanence de surfaces à pâturer de qualité ; pour cela fauchage ou gyrobroyage des excédents ou des refus sont nécessaires. À un tel chargement, on observe qu'il est possible d'adopter une conduite fourragère assurant un pâturage annuel quasi-continu. En outre, les mises bas de contre-saison étant limitées, il n'est plus indispensable d'avoir des bâtiments spécifiques d'agnelage et d'engraissement, ce qui limite les charges de structure. En définitive, avec des frais fourragers réduits de moitié et des frais de troupeaux réduits d'un 
tiers par rapport à ceux des exploitations herbagères autonomes caractérisées (groupe 3 ), les extensives très autonomes (groupe 2) bien gérées obtiennent une marge par brebis inférieure de $8 \%$ seulement à celle que les premières obtiennent et que l'on peut considérer comme optimale, à condition que la productivité numérique reste comprise entre 115 et $130 \%$.

\subsection{Forces et limites des élevages extensifs}

La technicité est de mise, la réussite des systèmes peu chargés réside dans la réduction des charges tout en maintenant la productivité animale à un niveau correct [8]. À l'utilisation des leviers techniques traditionnels (azote, aliments concentrés) doivent se substituer des conduites adaptées aux milieux, nécessitant plus d'observation, d'anticipation, de vue à long terme et de savoir faire. Parmi les dix élevages dont le chargement est inférieur à 0,85 (figure 3), six ont une autonomie fourragère inférieure à $75 \%$, avec des marges ovines médiocres dont trois ont des autonomies inférieures à $60 \%$. Ces derniers ont conservé des techniques et schémas classiques, et n'ont pas su tirer parti de la spécificité de chargements faibles.

Compte tenu de l'importance de la surface à entretenir (plus vaste à cheptel identique), nous avons pu souligner l'obstacle que représentent les charges foncières qui, ramenées à la brebis (ou à l'unité structurelle), peuvent s'avérer très lourdes.

Le rapport entre le revenu et le produit global est généralement supérieur en élevage extensif, ces systèmes ayant, d'une façon globale, recours à moins de charges. Un même revenu peut ainsi être obtenu par un produit inférieur. Le capital d'exploitation (hors foncier) est plus faible pour un même revenu. Dans les exploitations extensives très autonomes le rapport entre le capital d'exploitation (hors foncier) et le revenu est de 4 (et atteint 1,8 dans un cas), alors qu'il est de $7 \mathrm{chez}$ les intensives.

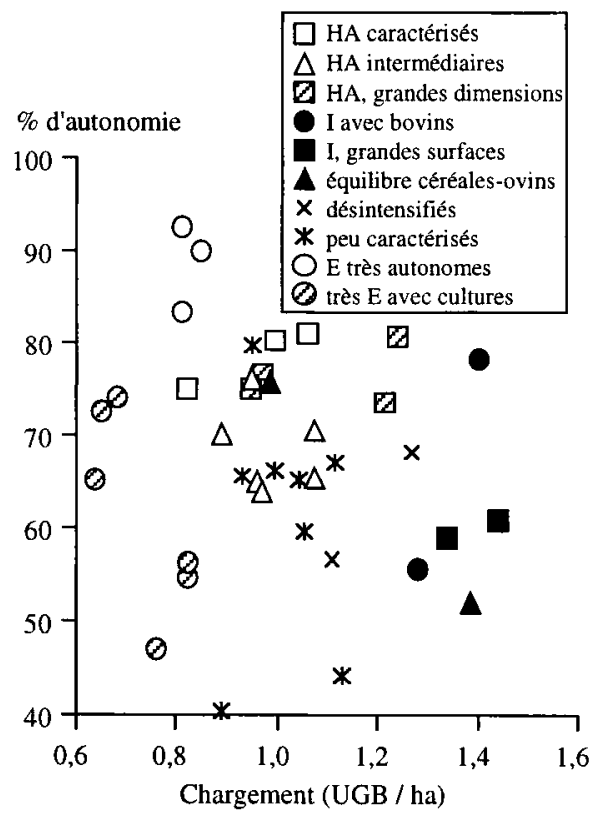

Figure 3. Relation entre l'autonomie fourragère et le chargement selon les types d'exploitations (HA, herbagers autonomes ; I, intensifs ; E, extensif). 


\section{CONCLUSION}

Les limites des systèmes d'élevage ovin viande intensifs apparues à la fin des années 80 ont été, dans un contexte de conjoncture très défavorable, accentuées par la réforme de la PAC de 1993 [1, 2]. Celle-ci, au travers des seuils techniques de versement d'aides (prime à l'herbe essentiellement), a contribué à réorienter les élevages intensifs des zones défavorisées - soit vers l'abandon partiel ou total de l'élevage des herbivores allaitants (ovins en premier lieu) au profit des cultures, en particulier les oléagineux et le maïs irrigué, - soit vers des systèmes à plus faible chargement (avec également l'attrait des cultures primées).

Dans les situations où l'on a observé une baisse relative du chargement (entre 1,0 et 1,1 ), celle-ci a généralement coïncidé avec une amélioration de l'autonomie fourragère et des résultats économiques. Cependant, des passages à des systèmes à moins de $0,90 \mathrm{UGB} \cdot \mathrm{ha}^{-1} \mathrm{n}$ 'ont pas toujours été réalisés avec une remise en cause des techniques classiques, en particulier la forte diminution nécessaire des charges et la mise en place de nouveaux itinéraires techniques.

Les conclusions des expérimentations systèmes de l'Inra de Clermont-Theix [8] et du LPA de Montmorillon [6] montrant que, dans ces contextes expérimentaux, l'amélioration des résultats économiques résultait du maintien du produit et d'une baisse des charges, n'ont été retrouvées qu'en partie : les exploitations extensives ayant les meilleures marges brutes par brebis ont opéré une diminution drastique des charges compensant une productivité du troupeau inférieure à la moyenne des exploitations de référence.

Les systèmes d'élevage extensifs originaux, identifiés lors de ce travail, peuvent ainsi être au départ de nouvelles réflexions, concernant plus particulièrement la façon de maximiser l'utilisation du pâturage en conservant une productivité animale minimale.

\section{RÉFÉRENCES}

[1] Benoit M., Laignel G., Liénard G., Fragilité des élevages ovins face à la baisse des prix. Réforme de la PAC et impact de la prime à l'herbe. Exemple du Montmorillonnais et du Massif Central Nord, Actes et Communications, $\mathrm{n}^{\circ} 10$, 145-I66, Inra-ESR, Paris, 1993.

[2] Benoit M., Laignel G., Liénard G., Adaptation des exploitations ovines du Montmorillonnais face à la réforme de la PAC. Premières années 1993 et 1994, Public. Lab. Économie Élevage, Inra, Theix, 1996, $31 \mathrm{p}$.

[3] Benoit M., Laignel G., Liénard G., Dedieu M., Chabosseau J.M., Éléments de réussite économique des élevages ovins extensifs du Montmorillonnais, Inra Prod. Anim. 10 (1997) 349-362.

[4] Béranger C., Extensification, l'évolution des réflexions; les acquis et les problèmes, in : L'extensification, une forme de modernisation. Séminaire de Dijon, Octobre 1990, Cifar, Paris, 1990, pp. 13-18.

[5] Chabosseau J.M., Laignel G., Lamoureux J., Souille C., Bergeron C., Staub A. 1996. Recherche sur les systèmes ovins herbagers en Montmorillonnais : étude de la conduite de 2 unités expérimentales chargées à 5 et 8 brebis/ha. Bilan 1995/1996, Doc. GIS Adaptation des exploitations du Montmorillonnais, $54 \mathrm{p}$.

[6] Chabosseau J.M., Dedieu B., La prairie temporaire et les interventions mécaniques en système ovin extensif : exemples d'élevages en Montmorillonnais, Fourrages 151 (1997) 351-372.

[7] Dedieu B., Chabosseau J.M., Benoit M., Laignel G, L'élevage ovin extensif du Montmorillonnais entre recherche d'autonomie, exigence des filières et simplicité de conduite, Inra Prod. animales 10 (1997) 207-218.

[8] Thériez M., Brelurut A., Pailleux J.Y., Benoit M., Liénard G., Louault F., De Montard F.X., Extensification en élevage ovin viande par agrandissement des surfaces fourragères, Inra Prod. Anim. 10 (2) (1997) 141-152. 